\title{
Point of care ultrasound in disaster triage
}

\author{
Brandon Chau, Gayathri Sivakumar \\ Faculty Reviewer: Robert Arntfield, MD, FRCPC, FCCP, FACEP (Division of Critical Care Medicine)
}

\begin{abstract}
Ultrasound technology has rapidly progressed over the past decades to emerge as a portable, versatile imaging modality to complement the physical exam. It has been shown to be superior to clinical exam and equivalent to computed-tomography in detecting many life-threatening conditions, and thus is used in a variety of resuscitative settings, including the front lines of the military. A variety of imaging protocols exist for ultrasound, but in resource-deplete settings such as a disaster scenario, the focused assessment with sonography for trauma (FAST) exam is a rapid and accurate method of determining acute intraperitoneal bleeding. With improving portability, as well as the ability to transmit images to a centralized command hub, it can become a key component of the first responder's toolkit.
\end{abstract}

\section{INTRODUCTION}

It wasn't so long ago that physicians began looking into the human body with ultrasound. In fact, ultrasound has barely been in the medical world for 60 years, initially to look at hearts, gallstones, and fetuses. ${ }^{1}$ As improvements in image resolution and miniaturization have taken place, ultrasound has similarly grown in leaps and bounds to the headline-making tool that it is today.

With miniaturization of the machines and the training of frontline clinicians in its use, ultrasound has left the confines of the radiology department and is now widely used in the portable, pointof-care setting. Point-of-care ultrasound (POCUS) is frequently used to guide bedside procedures such as central venous access, thoracentesis, and paracentesis. Diagnostically, it guides decision-making in cardio-respiratory failure and can identify ruptured abdominal aneurysms and triage those with first trimester bleeding. In the emergency department, the focused assessment with sonography for trauma (FAST) has become ubiquitous as a rapid and accurate way to rule out torso hemorrhage. ${ }^{2}$

With improving portability and accessibility, POCUS has a number of features that lends itself to utility in diverse locations such as high altitude, refugee camps, military combat, and even the International Space Station. ${ }^{2}$ Of particular interest, mass casualty incidents are an area where ultrasound offers an ideal pairing of portability and diagnostic power.

\section{ULTRASOUND USE IN THE MASS CASUALTY INCIDENT}

A mass casualty incident is defined as having two or more patients injured such that the medical need is not satisfied by available resources, whether it be the personnel, materials, or transport. ${ }^{3}$ In this scenario, priority rapidly shifts from providing best possible medical care to all patients to triaging resources to maximize the number of lives saved.

Multiple triage systems exist to determine priority of treatment, but they are all variations on a single theme. The Canadian Triage and Acuity Scale is often used in emergency departments, and uses slightly differing criteria to classify patients into five categories in order of descending urgency. ${ }^{4}$ Life-threatening events such as unstable trauma or cardiac arrest receive immediate attention, while medical urgencies such as "abdominal pain" and "shortness of breath" may be triaged to a lower category of urgency. ${ }^{4}$ Thus, triage serves to maximize the impact of the trained personnel when demand is in excess of its immediate supply.

\section{IN ONE OBSERVATIONAL STUDY,} ULTRASOUND RESULTS INFLUENCED OVER 7O\% OF PHYSICIAN DECISIONS OVER A 12-DAY SPAN.

The existing triage systems are not perfect. Over-triaging, resource over-allocation to patients who turn out to have minor medical ailments, and under-triaging are difficulties with an estimated occurrence of about $10 \%$ for each. ${ }^{2}$ It is possible that the dependence on history and physical examination, in the absence of medical imaging, contribute to this error. In the world of limited medical resources, such as military environments, POCUS has been demonstrated to be equally accurate to computed tomography (CT) scans in diagnosing pericardial effusion, pneumothorax and intraperitoneal fluid. ${ }^{2}$ Therefore, by incorporating an imaging modality reported to have sensitivity and specificity of up to $100 \%$ in identifying life-threatening conditions such as abdominal fluid, the rates of over- and under-triaging would likely decrease.

\section{REAL-WORLD EXAMPLES}

After the 2010 Haiti earthquake, POCUS was used by humanitarian workers to help triage patients presenting to local clinics. Specifically, ultrasound was used to diagnose conditions varying from pregnancy to hemothorax. In one observational study, ultrasound results influenced over $70 \%$ of physician decisions over a 12 day span. ${ }^{3}$

The value of ultrasound in austere environments has similarly been validated in the 1998 Armenian earthquake and the 2008 Wenchuan earthquake. Sensitivity and specificity of ultrasound-guided clinical diagnoses were $91.9 \%$ and $96.6 \%$, respectively. Finally, POCUS has been used in military deployments and reported to demonstrate high specificity (99\%) and sensitivity (100\%) in diagnosing pericardial/intraperitoneal fluid from both blunt and penetrating trauma as later confirmed by CT scans. ${ }^{2}$ 


\section{THE FAST EXAM}

Abdominal bleeding was historically diagnosed by the diagnostic peritoneal lavage, where a sterile solution was injected into the abdominal cavity and inspected for blood on drainage, or by CT scan. However, lavage is difficult in some patients and can be oversensitive, and the CT scan cannot be performed on an unstable patient. The FAST exam is a vast improvement on both these methods and can be completed in seconds to minutes by a trained clinician who can immediately integrate the findings into the management plan. It entails directly imaging the heart for evidence of hemopericardium, as well as imaging of the hepatorenal, splenic, and pelvic spaces for free intraperitoneal bleeding. ${ }^{5}$

A properly performed FAST exam with a negative finding can be sufficiently completed in 2.5 minutes, while an examination yielding a positive finding can be completed in mere seconds (a single positive finding is sufficient for escalation of medical attention). ${ }^{2}$ Furthermore, studies have demonstrated that even after a 1-day training course, ultrasound operators from varying levels of pre-existing expertise can obtain adequate images on ultrasound. ${ }^{2}$

\section{THE EFAST AND CAVEAT PROTOCOLS}

For all its benefits, the FAST exam is somewhat limited as it focuses on detecting abdominal and cardiac abnormalities, and does not scan for pneumothorax, which may be present in up to $20 \%$ of trauma patients. In the 1990's, the extended focused assessment in sonography for trauma (eFAST) protocol was introduced, expanding the FAST protocol to include thoracic imaging and searching for several signs to rule out pneumothorax. A 2004 study showed eFAST to be both more sensitive and specific than chest $\mathrm{x}$-rays in detecting pneumothorax. In fact, a recent study demonstrated that chest $\mathrm{x}$-rays can be omitted in lieu of the eFAST protocol in stable trauma patients, as the chest x-ray was found to have no benefit! ${ }^{6,7}$

The Chest Abdomen Vascular Extremities in Acute Triage (CAVEAT) protocol was proposed in 2010 as an expansion on the FAST and eFAST exams, as it can be used to detect other life-threatening conditions including long bone fractures and intravascular depletion. The CAVEAT protocol is a staged examination that begins with an eFAST exam, and, should time allow, moves on to examine vascular status by imaging the inferior vena cava for collapsibility, and the extremities. ${ }^{2}$ Validation studies continue to be pending for these expansions on the FAST examination, but studies continue to demonstrate promising results suggesting the effectiveness of POCUS.

\section{FUTURE OF ULTRASOUND IN DISASTER MEDICINE}

POCUS continues to have expansive uptake by providers as portability and affordability increase. Coupled with continuing validation of exam models such as eFAST and CAVEAT, there will likely be increasing inclusion of ultrasound in day-to-day patient care, particularly in the trauma setting.

In 2010, student innovators at Rice University in Houston, Texas developed a portable diagnostic kit dubbed "Lab-in-a-backpack". ${ }^{8}$ They have used this to tremendous success in humanitarian aid since then. With continued optimization of ultrasound technology, and improved portability, the POCUS exam can conceivably be combined with portable kits such as the lab-in-a-backpack.

\section{SUMMARY}

Once relegated to the dark rooms of the hospital, point-of-care ultrasound has burst onto the scene as a more global medical utility. In hospitals, ultrasound is used for diagnosis as well as for real-time guidance of invasive procedures. Improving portability and miniaturization of the technology has similarly rapidly expanded usability and versatility of ultrasound. Furthermore, ultrasound has proven to be an invaluable addition in the resource-depleted setting, as it has been shown to provide similar sensitivity and specificity as CT scans, at a fraction of the time and monetary cost.

Where the FAST exam has proven incredibly useful in diagnosing intra-abdominal fluid collection, new protocols such as eFAST and CAVEAT are expansions that systemically rule out other life-threatening concerns such as pneumothorax, long bone fractures and intravascular hypovolemia. In coming years, as techniques improve and technology continues to advance, the portable ultrasound will likely become a staple of every medical responder's toolkit.

\section{REFERENCES}

1. Woo J. A short History of the development of Ultrasound in Obstetrics and Gynecology. Ob-ultrasound.net. 2002. Accessed on Dec 5, 2016 at http://www.ob-ultrasound.net/historyl.html.

2. Wydo SM, Seamon MJ, Melanson SW, et al. Portable ultrasound in disaster triage: a focused review. Eur J Trauma Emerg Surg. 2016;42:1519.

3. Sajed D. The History of Point-of-Care Ultrasound Use in Disaster and Mass Casualty Incidents. Virtual Mentor. 2010;12(9):744-9.

4. Beveridge J, Clarke B, Janes L, et al. Implementation Guidelines. Canadian Association of Emergency Physicians. 2016. Accessed on Dec 5, 2016, at http://caep.ca/resources/ctas/implementation-guidelines.

5. Jang T. Focused Assessment with Sonography in Trauma (FAST). Medscape. 2015. Accessed on Dec 5, 2016, at http://emedicine.medscape.com/article/104363-overview.

6. Hamada SR, Delhaye N, Kerever S, et al. Integrating eFAST in the initial management of stable trauma patients: the end of plain film radiography. Ann Int Care. 2016;6:62.

7. Kirkpatrick AW, Sirois M, Laupland KB, et al. Hand-Held Thoracic Sonography for Detecting Post-Traumatic Pneumothraces: The Extended Focused Assessment With Sonography For Trauma (EFAST). J Trauma 2004;57:288-95.

8. Williams M. Lab-in-a-Backpack goes to work in Ecuador. RICE University News. 2010 Jan 3. Accessed on Dec 10, 2016 at http://news.rice. edu/2010/01/13/lab-in-a-backpack-goes-to-work-in-ecuador/. 